\title{
Improving the oxidation resistance of Inconel 718 high-temperature nickel alloy using combined surface engineering technology
}

\author{
Ph. V. Kiryukhantsev-Korneev ${ }^{\dagger, 1}$, A. E. Kudryashov ${ }^{1}$, A. N. Sheveyko ${ }^{1}$, \\ A.S. Orekhov ${ }^{1,2}$, E. A. Levashov ${ }^{1}$ \\ †kiruhancev-korneev@yandex.ru
}

\begin{abstract}
${ }^{1}$ National University of Science and Technology “MISiS”, 4 Leninskiy Pr., Moscow, 119049, Russia
${ }^{2}$ Federal Scientific Research Centre “Crystallography and Photonics”, RAS, 59 Leninsky Pr., Moscow, 119333, Russia
\end{abstract}

\begin{abstract}
To increase the oxidation resistance of the Inconel $718 \mathrm{Ni}$ alloy, a hybrid technology is proposed that combines electro-spark alloying (ESA) and magnetron sputtering (MS). Multiphase $\mathrm{ZrSiB}$ (ESA), ZrSiB, MoSiB, and CrAlSiB (MS) electrodes were obtained by self-propagating high-temperature synthesis. The study of the structure, composition and properties of singleand double-layers has been carried out. $\mathrm{ZrB}_{2}$ (73 wt.\%), $\mathrm{Si}(21 \mathrm{wt} . \%)$, Ni and $\mathrm{NiSi}_{x}$ traces (<5 wt.\%) were detected in the ESAlayer. The ESA-layer had a hardness of $19 \mathrm{GPa}$ and elastic modulus of $351 \mathrm{GPa}$. The $\mathrm{ZrSiB}$ MS-layer contained a h-ZrB ${ }_{2}$ phase with a 4-17 nm crystallites and Si-based amorphous regions. The layer hardness and elastic modulus were equal to 22 and $256 \mathrm{GPa}$, respectively. The MoSiB MS-layer had a conical structure, contained the h-MoSi ${ }_{2}$ and B-based amorphous phases. The layer showed hardness of $27 \mathrm{GPa}$ and elastic modulus of $389 \mathrm{GPa}$. $\mathrm{The}_{2} \mathrm{Cr}_{2} \mathrm{Al}, \mathrm{CrB}_{x}$, and $\mathrm{CrSi}_{2}$ phases were found in the CrAlSiB MS-layer with hardness of $19 \mathrm{GPa}$ and elastic modulus of $260 \mathrm{GPa}$. The $4.8 \mu \mathrm{m}$ oxide film was formed on the surface of the $\mathrm{Ni}$ alloy after annealing in air at $900^{\circ} \mathrm{C}$. The depth of the oxidation for $\mathrm{ZrSiB} / \mathrm{ZrSiB}, \mathrm{MoSiB} / \mathrm{ZrSiB}, \mathrm{and} \mathrm{CrAlSiB} / \mathrm{ZrSiB}$ MS-ESA coatings was 9.2, 0.4, and $0.12 \mu \mathrm{m}$, correspondingly. The ESA-layer acts as barrier to reduce the diffusion of elements from the substrate. Two-layer $\mathrm{MoSiB} / \mathrm{ZrSiB}$ and $\mathrm{CrAlSiB} / \mathrm{ZrSiB}$ coatings increase the oxidation resistance of the Ni alloy by 12 and 40 times, respectively, and can be recommended for deposition onto aircraft engine parts.
\end{abstract}

Keywords: electro-spark alloying, magnetron sputtering, self-propagating high-temperature synthesis, nickel alloy, oxidation resistance, coatings.

УДК: 621.762:620.22:621.9.048:621.793.182

\section{Повышение окислительной стойкости жаропрочного никелевого сплава ЭП-718 ИД с помощью комбинированной технологии инженерии поверхности}

\author{
Кирюханцев-Корнеев Ф. В. ${ }^{\dagger, 1}$, Кудряшов А.Е. ${ }^{1}$, Шевейко А. Н. ${ }^{1}$, \\ Орехов А.С. ${ }^{1,2}$, Левашов Е. А. ${ }^{1}$ \\ ${ }^{1}$ Национальный исследовательский технологический университет «МИСиС», \\ Ленинский пр., 4, Москва, 119049, Россия \\ ${ }^{2}$ Федеральный научно-исследовательский центр «Кристаллография и фотоника» РАН, \\ Ленинский пр., 59, Москва, 119333, Россия
}

Для повышения жаростойкости никелевого сплава ЭП-718 ИД (Inconel 718) предложен комбинированный способ нанесения покрытий, сочетающий технологии электроискрового легирования (ЭИЛ) и магнетронного напыления $(\mathrm{MH})$. В качестве электродов применяли многофазные материалы составов $\mathrm{ZrSiB}$ (для технологии ЭИЛ), $\mathrm{ZrSiB}$, $\mathrm{MoSiB}, \mathrm{CrAlSiB}$ (для MH), полученные методом самораспространяющегося высокотемпературного синтеза. Проведен комплекс исследований структуры, состава и свойств однослойных и комбинированных покрытий. В ЭИЛ 
слое выявлены фазы ZrB $(73$ вес.\%), Si (21 вес.\%), следы (<5 вес.\%) Ni и NiSi. ЭИЛ слой характеризуется твердостью 19 ГПа и модулем упругости 351 ГПа. Основу слоя $\mathrm{ZrSiB}$, полученного MH, составляла гексагональная фаза h-ZrB 2 с размером кристаллитов 4-17 нм. Кремний находился в аморфных областях. Твёрдость слоя - 22 ГПа, модуль упругости - 256 ГПа. MH-слой MoSiB имел выраженную конусную структуру. Основой являлась фаза h-MoSi 2 , также присутствовал аморфный МоВ. Слой характеризовался твёрдостью 27 ГПа и модулем упругости 389 ГПа. В МН-слое CrAlSiB обнаружены фазы - $\mathrm{Cr}_{2} \mathrm{Al}, \mathrm{CrB}_{x^{\prime}}, \mathrm{CrSi}_{2}$, твёрдость слоя - 19 ГПа, модуль упругости - 260 ГПа. В результате высокотемпературного воздействия при $900^{\circ} \mathrm{C}$ на поверхности сплава ЭП-718ИД образовалась оксидная пленка толщиной 4.8 мкм. Глубина окисленного слоя у двухслойных MH-ЭИЛ покрытий $\mathrm{ZrSiB} / \mathrm{ZrSiB}$ составляла 9.2 мкм, $\mathrm{MoSiB} / \mathrm{ZrSiB}$ - 0.4 мкм, CrAlSiB/ZrSiB - 0.12 мкм. Установлена барьерная роль ЭИЛ-подслоя, снижающая диффузию элементов материала подложки. Двухслойные покрытия MoSiB/ZrSiB и CrAlSiB/ZrSiB повышают жаростойкость никелевого сплава, соответственно в 12 и в 40 раз и могут быть рекомендованы для обработки деталей авиационных двигателей из никелевого сплава ЭП-718 ИД.

Ключевые слова: электроискровое легирование, магнетронное напыление, самораспространяющийся высокотемпературный синтез, никелевый сплав, жаростойкость, покрытия.

\section{1. Введение}

Жаропрочные материалы на никелевой основе находят широкое применение в конструкциях авиационных, морских и наземных газотурбинных двигателей в качестве деталей горячего тракта: лопаток турбин и компрессоров, дисков турбин, жаровых труб, камер сгорания, корпусных деталей. Никелевые жаропрочные сплавы успешно применяются в энергетической (энергоустановках на базе газотурбинных двигателей), ядерной и стекольной промышленностях [1-3]. Разработки в области жаропрочных сплавов и сталей проводят компании России, ЕС, США, Японии, Швейцарии, Китая, Индии и других стран [4].

Одним из эффективных способов повышения ресурса работы и эксплуатационных характеристик жаропрочных сплавов является применение защитных покрытий $[5,6]$. К наиболее распространенным методам осаждения покрытий относятся электроискровое легирование (ЭИЛ) [7] и магнетронное напыление (МН) [8].

Несмотря на преимущества метода ЭИЛ (высокая адгезия, простота реализации, низкая энергоемкость и высокая экологичность), покрытия, нанесенные даже в автоматизированном режиме, имеют высокий уровень дефектов (поры, микротрещины) и повышенную шероховатость поверхности. В связи с этим актуально сочетать ЭИЛ с другими методами инженерии поверхности. Известны работы по вторичной обработке ЭИЛ-слоя методами микродугового оксидирования [9], лазерного оплавления [10], диффузионного насыщения [11]. Процесс ЭИЛ может сочетаться с ионно-плазменным напылением [12]. Ионно-плазменное осаждение позволяет залечить дефекты поверхности ЭИЛ-покрытий [13], что особенно важно для предотвращения катастрофического окисления ответственных деталей и узлов [14].

В качестве электродных материалов для ЭИЛ применяют металлы и их сплавы, графит, твердые сплавы, а также керамические материалы, полученные методом самораспространяющегося высокотемпературного синтеза (CBC) [15]. СBC-электроды CrAlSiB, MoSiB и $\mathrm{ZrSiB}$ успешно апробированы для создания ЭИЛ [16-18] и МН [19-21] жаростойких покрытий.

В представленном исследовании для повышения эксплуатационных свойств жаропрочного никелевого сплава ЭП718-ИД (Inconel 718), предложен комбинированный способ нанесения покрытий: на первом этапе поверхность обрабатывается с помощью ЭИЛтехнологии электродом $\mathrm{ZrSiB}$, а на втором этапе на сформированный слой осаждается покрытие путем магнетронного распыления СВС-мишени одного из составов $\mathrm{ZrSiB}, \mathrm{MoSiB}$ или CrAlSiB. Целью данной работы является изучение структуры и свойств комбинированных МН-ЭИЛ покрытий на никелевом сплаве ЭП-718ИД, а также получение эффективных покрытий для практического применения.

\section{2. Методика эксперимента}

Керамические материалы для изготовления электродов были получены с помощью СВС-компактирования смесей элементных порошков. Заготовки для придания необходимой формы подвергались электроэрозионной резке и обработке на плоско-шлифовальном станке. На стадии ЭИЛ обработки использовался СВСэлектрод $\mathrm{ZrSiB}$ (вес.\%: $66 \mathrm{ZrB}_{2}, 26 \mathrm{ZrSi}_{2}, 6 \mathrm{Si}, 2 \mathrm{ZrO}_{2}$ ) размером $4 \times 4 \times 50$ мм. На стадии МН использовались CBC-мишени $\mathrm{ZrSiB}$ (аналогичный состав), CrAlSiB (вес.\%: $62 \mathrm{CrB}, 33$ (Cr, Al) $\mathrm{Si}_{2}, 5 \mathrm{Cr}_{5} \mathrm{Si}_{3} \mathrm{~B}_{x}$ ) и $\mathrm{MoSiB}$ (вес.\%: $\left.89 \mathrm{MoSi}_{2}, 11 \mathrm{MoB}\right)$ в форме дисков $\varnothing 120 \times 10$ мм. Покрытия осаждались на никелевый сплав ЭП718-ИД (ТУ 14-1-3905-85), широко применяемый в авиационном двигателестроении. Размер подложек составлял $\varnothing 28 \times 5$ мм, площадь обрабатываемой поверхности 6 см² $^{2}$

ЭИЛ-обработка проводилась в Ar на установке Alier 303 Metal при прямой полярности (анод - CBCэлектрод, катод - подложка), силе тока в разряде $120 \mathrm{~A}$, частоте импульсов 3200 Гц, длительности импульса 20 мкс. Для МН применялась установка УВН-2М, оснащённая магнетроном и ионным источником. С помощью блока питания Advanced Energy Pinnacle+ на магнетрон подавалась мощность 1 кВт, ток составлял 2 А. Дистанция между мишенью и подложкой составляла 8 см, время осаждения 40 мин, среда $\operatorname{Ar}$ (99.9995\%), остаточное/рабочее давление 0.001/0.2 Па. Были получены двухслойные ЭИЛ-МН покрытия, а для сравнения - однослойные ЭИЛ- и МН-покрытия.

Шлифы полировались на машине RotoPol-21 Struers в автоматическом режиме. Микроструктура исследова- 
лась на сканирующем электронном микроскопе (СЭМ) (Hitachi S-3400N) с приставкой для энергодисперсионной спектроскопии (ЭДС) (NORAN 7). Элементные профили получены методом оптической эмиссионной спектроскопии тлеющего разряда (ОЭСТР) (Profiler 2 Horiba JY). Исследования тонкой структуры выполнены на просвечивающем электронном микроскопе (ПЭМ) JEM-2100 Jeol. Фольги готовились на комплексе пробоподготовки с установками для ионно-лучевого травления PIPS II System (Gatan, CША) и FIB (FEI Quanta 2003D FIB instrument, США). Рентгенофазовый анализ (РФА) покрытий проводили на дифрактометрах ДРОН-4 и Bruker-D8 с использованием монохроматического $\mathrm{Cu}_{\text {Ка }}$-излучения.

Механические свойства определялись методом наноиндентирования (NanohardnessTester, CSM Instr.). Испытания на жаростойкость выполнены в электропечи SNOL 7.2/1200 при температуре $900^{\circ} \mathrm{C}$ и выдержке 5 ч. После отжига проводились ОЭСТР и СЭМ-ЭДС исследования. За глубину окисления $\left(h_{\text {ох }}\right)$ принималась толщина поверхностного слоя, на которой содержание О превышало 10 ат.\%. Оценка жаростойкости весовым методом проводилась путём взвешивания образцов до и после отжига на аналитических весах KERN 770 с точностью $10^{-5}$ г.

\section{3. Результаты и их обсуждение}

ЭИЛ слои, сформированные электродом $\mathrm{ZrSiB}$, обладали высокой однородностью, сплошностью до 100\% и толщиной около 35 мкм (Рис. S1, дополнительный материал). По данным РФА основной фазой являлся диборид циркония (73 вес.\%) с размером зерна 1-4 мкм. В составе покрытия также присутствует Si (21 вес.\%) и малые концентрации (<5 вес.\%) Ni и $\mathrm{NiSi}_{x}$. Покрытие характеризуются твердостью 19 ГПа, модулем упругости 351 ГПа, невысокой шероховатостью $\left(R_{a}=5.8\right.$ мкм).

Основу слоёв $\mathrm{ZrSiB}$, полученных методом $\mathrm{MH}$, составляла гексагональная фаза $\mathrm{h}-\mathrm{ZrB}_{2}$ с размером кристаллитов от 4 до 17 нм. На электронограммах покрытий не было обнаружено линий, связанных с кристаллическими кремнийсодержащими фазами, что свидетельствует о том, что Si находится преимущественно в аморфных областях. Твёрдость и модуль упругости составляли 22 и 256 ГПа, соответственно. МН-слои $\mathrm{MoSiB}$ имели выраженную конусную структуру, формирующуюся после слоя с равноосной структурой толщиной около 400 нм. Столбчатые зёрна имели Ø5-25 нм вблизи подложки. Диаметр увеличивался по мере роста покрытия, достигая Ø90-220 нм. Согласно данным дифракции электронов основу покрытий составляла фаза h-MoSi ${ }_{2}$. Бор присутствовал в аморфной фазе, близкой по составу к МоВ. Слои $\mathrm{CrAlSiB}$, осаждённые методом MH, содержали несколько кристаллических фаз на основе соединений хрома, $\mathrm{Cr}_{2} \mathrm{Al}, \mathrm{CrB}_{x}, \mathrm{CrSi}_{2}$, что хорошо видно из темнопольных изображений структуры. MoSiB и CrAlSiB показали твёрдость и модуль упругости 27 и 19 ГПа, 389 и 260 ГПа, соответственно. Данные ПЭМ для $\mathrm{MH}$ слоёв представлены на Рис. S2 (дополнительный материал).
Состав различных областей двухслойного МН-ЭИЛ покрытия на примере системы $\mathrm{ZrSiB} / \mathrm{CrAlSiB}$ (Рис. 1) приведен в Табл. 1. В МН слое (область 1) обнаружены элементы, соответствующие материалу СВС-мишени. В ЭИЛ-подслое (область 2) наблюдаются элементы, входящие в состав как электродного материала (Zr, $\mathrm{Si}, \mathrm{B})$, так и в материал подложки ( $\mathrm{Ni}, \mathrm{Fe}, \mathrm{Cr}, \mathrm{Al}$ ). Наличие элементов ЭП-718 ИД в подслое связано с перемешиванием материалов электрода и подложки в процессе ЭИЛ. В области 3 выявлены элементы, входящие в состав никелевого сплава. Область 4, в которой присутствуют $\operatorname{Zr}(89.8$ вес.\%) и В (7.5 вес.\%), соответствует фазе $\mathrm{ZrB}_{2}$. Остальные двухслойные покрытия демонстрировали аналогичную структуру: верхний МН-слой с высокой плотностью и отсутствием дефектов, в котором структурные элементы не выявлялись и нижний ЭИЛ-слой, состоящий в основном из зёрен $\mathrm{ZrB}_{2}$ (>70 вес.\% по данным РФА) различной дисперсности, а также фаз на основе $\mathrm{Si}$ и $\mathrm{Ni}$.

Элементные ОЭСТР-профили одно- и двухслойных покрытий на примере пары MoSiB-ZrSiB представлены на Рис. 2. Однослойное ЭИЛ-покрытие $\mathrm{ZrSiB}$ демонстрирует плавное уменьшение концентрации элементов электрода $(\mathrm{Zr}, \mathrm{Si}, \mathrm{B})$ и одновременный рост содержания компонентов подложки ( $\mathrm{Ni}, \mathrm{Cr}, \mathrm{Fe}$ ) при увеличении глубины анализа. Выраженный градиент концентраций связан с физикой процесса ЭИЛ, в частности, с протеканием в зоне разряда металлургических реакций, при которых происходит перемешивание составляющих электродов. Подобная структура обеспечивает высокую

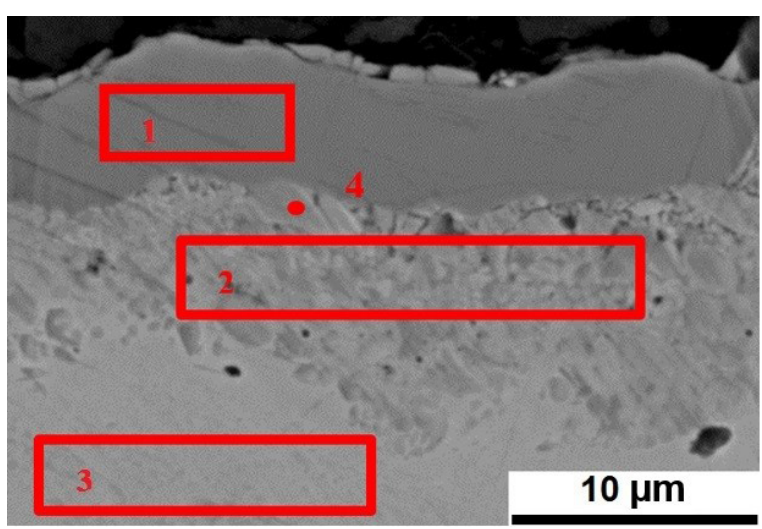

Рис. 1. Данные СЭМ для МН-ЭИЛ покрытия CrAlSiB/ZrSiB в исходном состоянии.

Fig. 1. SEM data for CrAlSiB/ZrSiB double-layer MS-ESA coating in the as-deposited state.

Табл. 1. Элементный состав (вес.\%) двухслойного МН-ЭИЛ покрытия $\mathrm{CrAlSiB} / \mathrm{ZrSiB}$ в разных областях по данным ЭДС (Рис. 1).

Table 1. Element composition (wt.\%) for CrAlSiB/ZrSiB double-layer MS-ESA coating in different areas according to the EDS (Fig. 1).

\begin{tabular}{|c|c|c|c|c|c|c|c|c|c|}
\hline № & $\mathrm{B}$ & $\mathrm{Al}$ & $\mathrm{Si}$ & $\mathrm{Ti}$ & $\mathrm{Cr}$ & $\mathrm{Fe}$ & $\mathrm{Ni}$ & $\mathrm{Zr}$ & $\mathrm{Mo}$ \\
\hline 1 & 2.2 & 9.5 & 14.2 & & 73.7 & 0.3 & 0.0 & 0.0 & \\
\hline 2 & 6.2 & 0.1 & 23.4 & & 3.3 & 5.6 & 11.3 & 50.1 & \\
\hline 3 & 0.0 & 1.2 & 1.5 & 2.2 & 16.5 & 27.3 & 47.6 & 0.0 & 3.8 \\
\hline 4 & 7.5 & 0.0 & 1.6 & & 0.0 & 0.2 & 1.0 & 89.8 & \\
\hline
\end{tabular}


адгезию [7]. Напротив, MH-покрытие MoSiB демонстрирует выраженную границу и резкий переход от элементов покрытия к элементам подложки, поскольку зона их химического взаимодействия крайне мала и не превышает толщину нескольких атомных слоёв. Двухслойные покрытия объединили особенности каждого типа покрытий и содержат: верхний МН-слой с постоянным по толщине составом и нижний несущий градиентный ЭИЛ-слой с плавным переходом к составу подложки.

Структура МН-ЭИЛ покрытия $\mathrm{CrAlSiB} / \mathrm{ZrSiB}$ после отжига представлена на Рис. 3. Составы областей указаны в Табл. 2. Для сравнения на Рис. S3 (дополнительный материал) приведены элементные карты до и после отжигов. Выявлено, что МН-слой герметизирует трещины, возникающие после ЭИЛ-обработки.

На поверхности МН-покрытия CrAlSiB установлено образование двух слоев. Кроме того, после окислительного отжига происходит взаимная диффузия элементов подложки (Ni, Fe, Cr) в МН-слой, а Zr из ЭИЛ-слоя в МН-слой. Кислород содержится преимущественно в поверхностном слое (область 2). В результате отжига в области 1, контактирующей с воздухом, по сравнению с аналогичной областью образца до отжига (Табл. 1), наблюдается снижение концентрации $\mathrm{Cr}, \mathrm{Al}$, Si и B. В области 2, находящейся на границе МН и ЭИЛ слоёв, содержатся $\mathrm{Ni}, \mathrm{Fe}$, а также $\mathrm{Zr}$ (элемент материала ЭИЛподслоя), а также О (4.8 вес.\%). В ЭИЛ-слое (область 3) обнаружено повышенное по сравнению с исходным состоянием содержание $\mathrm{Ni}$ (33.3 вес.\%), Fe (14.5 вес.\%) и $\mathrm{Cr}$ (6.6 вес.\%), а в подложке (область 4) - элементы, входящие в состав сплава. В области 6, находящейся на границе ЭИЛ-подслой-подложка, обнаружено

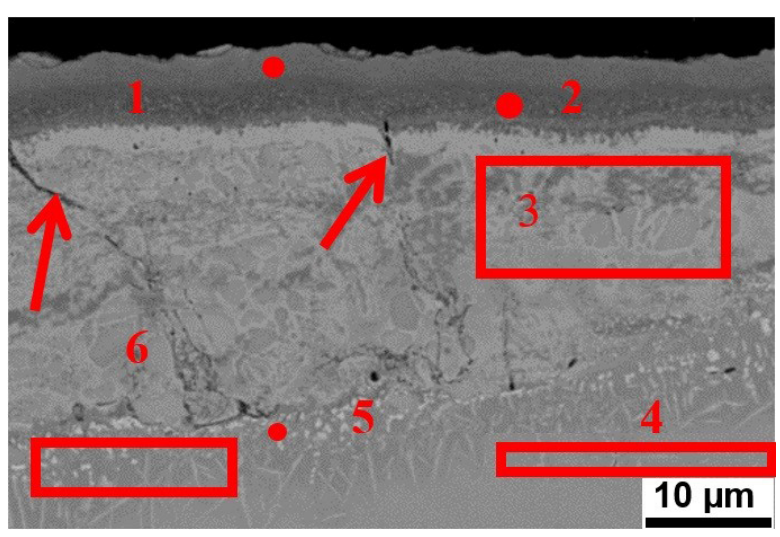

Рис. 3. Данные СЭМ для МН-ЭИЛ покрытия $\mathrm{CrAlSiB} / \mathrm{ZrSiB}$ после отжига.

Fig. 3. SEM data for CrAlSiB/ZrSiB double-layer MS-ESA coating after annealing.

повышенное содержание Si (6.6 вес.\%). В подложке содержится 0.3 вес.\% Si, поэтому можно предположить, что Si диффундирует в переходную область из ЭИЛ слоя. В области 5 отмечена повышенная концентрация Мо и W.

ОЭСТР-профили распределения элементов в двухслойных покрытиях после отжига приведены на Рис. 4. Глубина окисленного слоя в подложке, в одно- и двухслойных покрытиях, определённых ОЭСТР, представлена на Рис. 5. В результате отжига на поверхности образца без покрытия образовалась оксидная пленка толщиной 4.8 мкм, характеризующаяся низкой адгезией. Под оксидной пленкой отмечаются участки локального окисления. В результате отжига поверхностный слой обеднен $\mathrm{Cr}$ и обогащен $\mathrm{Ni}$ и $\mathrm{Fe}$.

Табл. 2. Элементный состав (вес.\%) двухслойного МН-ЭИЛ покрытия CrAlSiB/ZrSiB после отжига по данным ЭДС (Рис. 3).

Table 2. Element composition (wt.\%) for CrAlSiB/ZrSiB double-layer MS-ESA coating after annealing in different areas according to the EDS (Fig. 3).

\begin{tabular}{|c|c|c|c|c|c|c|c|c|c|c|c|c|}
\hline № & $\mathrm{B}$ & $\mathrm{O}$ & $\mathrm{Al}$ & $\mathrm{Si}$ & $\mathrm{Ti}$ & $\mathrm{Cr}$ & $\mathrm{Fe}$ & $\mathrm{Ni}$ & $\mathrm{Zr}$ & $\mathrm{Nb}$ & $\mathrm{Mo}$ & $\mathrm{W}$ \\
\hline 1 & 1.9 & 0.0 & 1.7 & 7.6 & & 46.0 & 9.8 & 32.5 & 0.6 & & & \\
\hline 2 & 3.6 & 4.8 & 8.1 & 3.5 & & 58.8 & 6.6 & 13.6 & 1.1 & & & \\
\hline 3 & 7.5 & 0.4 & 0.1 & 6.9 & & 6.6 & 14.5 & 33.3 & 30.6 & & & \\
\hline 4 & 0.0 & 0.0 & 1.3 & 0.0 & 2.3 & 15.6 & 29.1 & 43.7 & 0.0 & & 4.2 & 3.8 \\
\hline 5 & 0.8 & 0.0 & 0.3 & 8.5 & 5.7 & 6.1 & 14.5 & 36.7 & 0.0 & 2.9 & 12.8 & 11.6 \\
\hline 6 & 0.2 & 0.0 & 1.2 & 6.6 & 2.3 & 11.3 & 31.9 & 37.8 & 0.0 & 0.4 & 4.3 & 4.1 \\
\hline
\end{tabular}

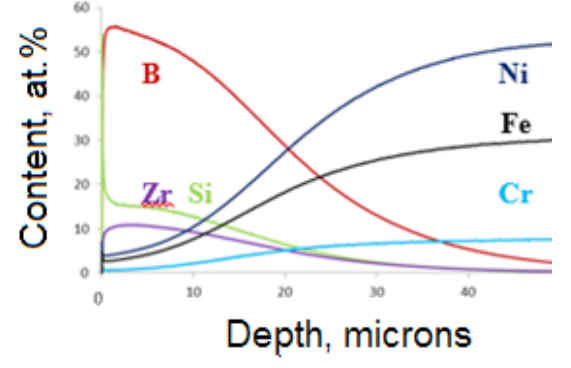

a

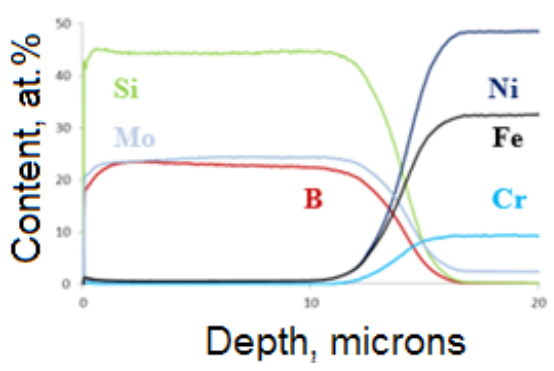

$\mathrm{b}$

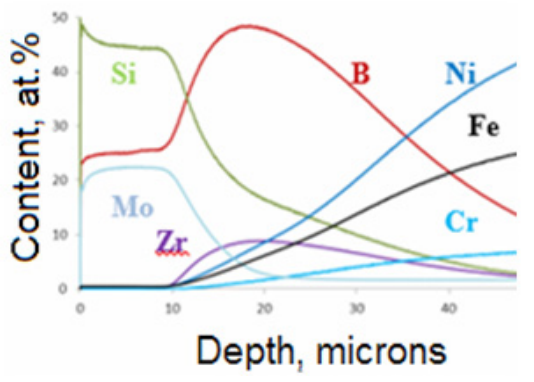

C

Pис. 2. (Color online) ОЭСТР-профили для однослойного ЭИЛ-покрытия $\mathrm{ZrSiB}$ (a), однослойного MH-покрытия MoSiB (b), двухслойного МН-ЭИЛ покрытия $\mathrm{MoSiB} / \mathrm{ZrSiB}(\mathrm{c})$.

Fig. 2. (Color online) GDOES-profiles for single-layer ZrSiB ESA-coating (a), single-layer MoSiB MS-coating (b), and double-layer $\mathrm{MoSiB} / \mathrm{ZrSiB}$ MS-ESA coating (c). 


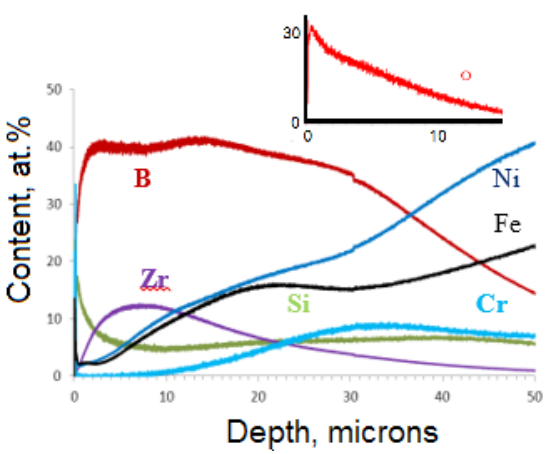

a

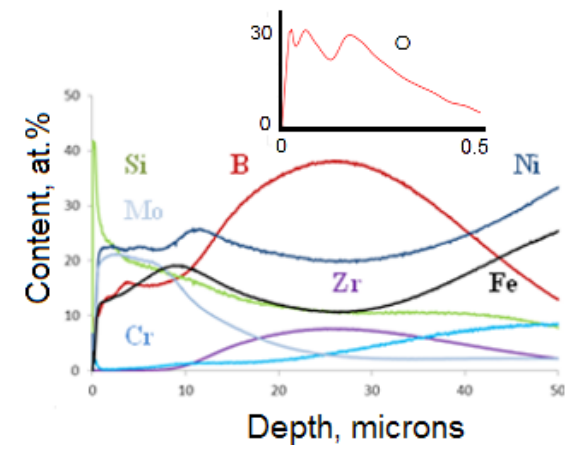

b

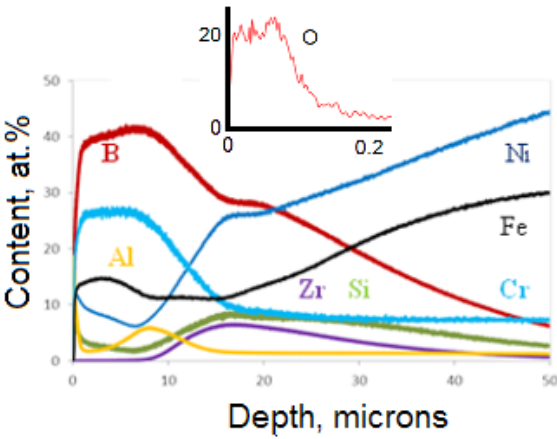

c

Pис. 4. (Color online) Обзорные ОЭСТР-профили и элементные профили кислорода для МН-ЭИЛ покрытий $\mathrm{ZrSiB} / \mathrm{ZrSiB}$ (a), $\mathrm{MoSiB} / \mathrm{ZrSiB}$ (b) и CrAlSiB/ZrSiB (c) после отжига.

Fig. 4. (Color online) Complex GDOES-profiles and oxygen profiles for $\mathrm{ZrSiB} / \mathrm{ZrSiB}$ (a), MoSiB/ZrSiB (b) и CrAlSiB/ZrSiB (c) MS-ESA coatings after annealing.

Толщина окисленного слоя под удаленной оксидной пленкой составляет 0.2 мкм. Значение $h_{\text {ох }}$ ЭИЛ покрытия $\mathrm{ZrSiB}$ составило 1.5 мкм, что по-видимому, связано с присутствием в поверхностном слое элементов подложки, в первую очередь $\mathrm{Ni}, \mathrm{Fe}$, которые преимущественно окисляются. Среди однослойных покрытий минимальной толщиной окисленного слоя 0.45 и 0.20 мкм характеризовались образцы с МН покрытиями $\mathrm{MoSiB}$ и CrAlSiB, соответственно. Глубина окисленного слоя для CrAlSiB не превышает 0.20 мкм, что в 7.5 раз меньше, чем значение для ЭИЛ-покрытия $\mathrm{ZrSiB}$. Предварительное нанесениие ЭИЛ-слоя ZrSiB способствует улучшению жаростойкости двухслойного покрытия, по сравнению с однослойным MH-покрытием ZrSiB. Величина $h_{\text {ох }}$ в этом случае составляет 9.2 мкм. Применение комбинированного МН-ЭИЛ метода способствует снижению $h_{\text {ох }}$ при использовании покрытий $\mathrm{MoSiB} / \mathrm{ZrSiB}$ до 0.40 мкм, а при применении CrAlSiB/ZrSiB до 0.12 мкм. Важно отметить, что наблюдаемая диффузия элементов подложки (особенно Ni) к поверхности образца при нагреве, менее всего выражена в случае покрытия CrAlSiB/ZrSiB.

На Рис. 5 приведено удельное изменение массы $(\Delta m / S)$ образцов с покрытиями после отжига. Установлено, что у $\mathrm{MH}$ покрытий $\mathrm{MoSiB}$ и CrAlSiB наблюдается убыль массы образца, что, по-видимому, связано с выгоранием бора. Максимальный привес выявлен у однослойного $\mathrm{MH}$-покрытия $\mathrm{ZrSiB}\left(\Delta m / S=1.05 \mathrm{мг} / \mathrm{cm}^{2}\right)$ и двухслойного МН-ЭИЛ покрытия $\mathrm{ZrSiB} / \mathrm{ZrSiB}$

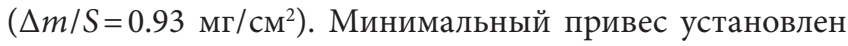
у двухслойных МН-ЭИЛ покрытий $\mathrm{MoSiB} / \mathrm{ZrSiB}$ $\left(\Delta m / S=0.06 \mathrm{мг} / \mathrm{cm}^{2}\right)$ и $\mathrm{CrAlSiB} / \mathrm{ZrSiB}\left(\Delta m / S=0.21 \mathrm{мг} / \mathrm{cm}^{2}\right)$. Это свидетельствует о наиболее высокой жаростойкости указанных комбинированных покрытий. Можно отметить, что привес ЭИЛ покрытия $\mathrm{ZrSiB}$ меньше, чем неупрочненного образца из никелевого сплава.

\section{4. Заключение}

Предложен комбинированный метод нанесения защитных покрытий на жаропрочный никелевый сплав ЭП-718ИД, состоящий из последовательно проводимых

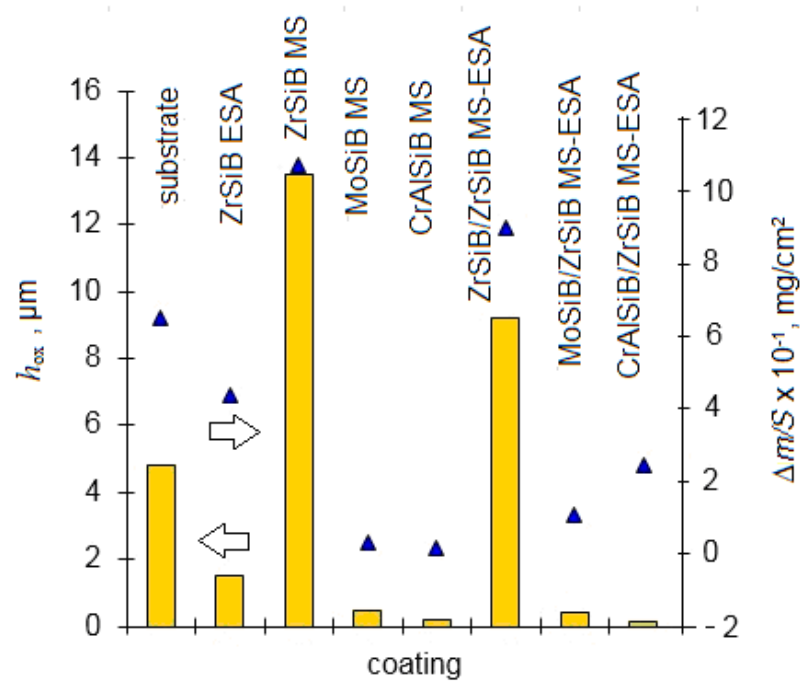

Pис. 5. (Color online) Глубина окисления $\left(h_{\text {ох }}\right)$ и удельное изменение массы $(\Delta m / S)$ образцов после высокотемпературного воздействия.

Fig. 5. (Color online) Oxidation depth $\left(h_{\mathrm{ox}}\right)$ and mass change $(\Delta m / S)$ of the samples after annealing.

операций электроискрового и магнетронного осаждения. Установлена барьерная роль ЭИЛ подслоя при длительном высокотемпературном окислении, снижающая диффузию элементов материала подложки в $\mathrm{MH}$-слой. Применение двухслойных покрытий $\mathrm{MoSiB} / \mathrm{ZrSiB}$ и $\mathrm{CrAlSiB} / \mathrm{ZrSiB}$ позволяет существенно увеличить жаростойкость никелевого сплава в 12 и в 40 раз, соответственно. Комбинированные МН-ЭИЛ покрытия $\mathrm{MoSiB} / \mathrm{ZrSiB}$ и $\mathrm{CrAlSiB} / \mathrm{ZrSiB}$ рекомендованы для обработки деталей авиационных двигателей из никелевого сплава ЭП-718 ИД.

Дополнительный материал / Supplementary material. Электронная версия статьи содержит дополнительный материал, доступный безвозмездно на сайте журнала (lettersonmaterials.com). / The online version of this paper contains supplementary material available free of charge at the journal's Web site (lettersonmaterials.com). 
Благодарности / Acknowledgements. Исследования методом просвечивающей электронной микроскопии выполнены в рамках государственного задания (проект № 0718-2020-0034). / This work was carried out with financial support from the Ministry of Science and Higher Education of the Russian Federation (Project No. 0718-2020-0034 of State assignment) in the part of TEM studies.

\section{Литература/References}

1. E. N. Kablov. Liteynoye proizvodstvo. 7, 2 (2005). (in Russian) [Е.Н. Каблов. Литейное производство. 7, 2 (2005).]

2. B. S. Lomberg, S. A. Moses. All the materials. Encyclopedic reference book. 6, 2 (2007). (in Russian) [Б. С. Ломберг, C.А. Моисеев. Все материалы. Энциклопедический справочник. 6, 2 (2007).]

3. O. A. Bazyleva, E. G. Arginbaeva, S. A. Lutsk. Proceedings of VIAM. 4 (64), 3 (2018). (in Russian) [О.А. Базылева, Э. Г. Аргинбаева, С. А. Луцкая. Труды ВИАМ. 4 (64), 3 (2018).] Crossref

4. O.G. Ospennikova. Aviation materials and technologies. S, 19 (2012). (in Russian) [О. Г. Оспенникова. АМИТ. S, 19 (2012).]

5. V.A. Poklad, Yu. P. Shkretov, N. V. Abraimov. Engine. 4 (70), 2 (2010). (in Russian) [В. А. Поклад, Ю. П.Шкретов, Н. В. Абраимов. Двигатель. 4 (70), 2 (2010).]

6. M. Khoking, V. Vasantasri, P. Sidki. Metallic and Ceramic Coatings: Manufacturing, Properties, and Application. Moscow, Mir (2000) 516 p. (in Russian) [М. Хокинг, В. Васантасри, П. Сидки. Металлические и керамические покрытия: Получение, свойства и применение (пер. с англ.). Москва, Мир (2000) 516 c.]

7. S.V. Nikolenko, A.D. Verkhoturov. New Electrode Materials for Electrospark Alloying. Vladivostok, Dal'nauka (2005) 217 p. (in Russian) [C. В. Николенко, А.Д. Верхотуров. Новые электродные материалы для электроискрового легирования. Владивосток, Дальнаука (2005) 217 с.]

8. D. A. Chubarov, S. A. Budinovsky, A. A. Smirnov. Aviation materials and technologies. 4 (45), 23 (2016). (in Russian)
[Д.А. Чубаров, С.А. Будиновский, А.А. Смирнов. АМИТ. 4 (45), 23 (2016).]

9. S. Durdu, S. L. Aktug, K. Korkmaz. Surf. Coat. Technol. 236, 303 (2013). Crossref

10. N. Radek, K. Bartkowiak. Phys. Procedia. 39, 295 (2012). Crossref

11. A. Bejar, W. Schnake, W. Saavedra, J.P. Vildósolaet. J. Mater. Process. Tech. 176, 210 (2006). Crossref

12. D. V. Shtansky, I. V. Batenina, I. A. Yadroitsev, N. S. Ryashin, Ph.V. Kiryukhantsev-Korneev, A.E. Kudryashov, A.N. Sheveyko, I.Y. Zhitnyak, N.A. Gloushankova, E. A. Levashov. Surf. Coat. Technol. 208, 14 (2012). $\underline{\text { Crossref }}$

13. Ph.V. Kiryukhantsev-Korneev, N.V. Shvyndina, A.D. Sytchenko, D.V. Shtansky, V.A. Gorshkov, E.A. Levashov. J. Phys.: Conf. Ser. 1431, 012027 (2020). Crossref

14. E.N. Kablov, S.A. Muboyajyan. Aviation materials and technologies. (in Russian) [Е.Н. Каблов, С. А. Мубояджян. АМИТ. 5, 60 (2012).]

15. Advanced Materials and Technologies for SelfPropagating High-Temperature Synthesis. Moscow, Publishing House MISiS. (in Russian) [Е.А. Левашов, А.С. Рогачев, В.В. Курбаткина, Ю.М. Максимов, В.И. Юхвид. Перспективные материалы и технологии самораспространяющегося высокотемпературного синтеза. Москва, Изд. Дом МИСиС (2011) 377 с.]

16. A.E. Kudryashov, A.Yu. Potanin, D.V. Shtansky, E. A. Levashov. Surf. Coat. Technol. 285, 278 (2016). Crossref

17. A.E. Kudryashov, D. N. Lebedev, A. Yu. Potanin. Surf. Coat. Technol. 335, 104 (2018). Crossref

18. A.E. Kudryashov, Ph.V. Kiryukhantsev-Korneev, M.I. Petrzhik, E. A. Levashov. CIS Iron and Steel Review. 18, 46 (2019). Crossref

19. Ph. V. Kiryukhantsev-Korneev, I. V. Iatsyuk, N. V. Shvyndina, E. A. Levashov, D. V. Shtansky. Corros. Sci. 123, 319 (2017). Crossref

20. Ph. V. Kiryukhantsev-Korneev, J.F. Pierson, K. A. Kuptsov, D. V. Shtansky. Appl. Surf. Sci. 314, 104 (2014). Crossref

21. Ph.V. Kiryukhantsev-Korneev, M.V. Lemesheva, N.V. Shvyndina, E.A. Levashov, A.Yu. Potanin. Prot. Met. Phys. Chem. 54 (6), 1147 (2018). ․ㅏossref 